\title{
A time reclustering algorithm for jet quenching studies
}

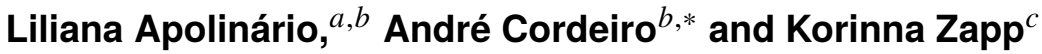 \\ ${ }^{a} L I P$, \\ Av. Prof. Gama Pinto, 2, P-1649-003 Lisbon, Portugal \\ ${ }^{b}$ Department of Physics, IST, University of Lisbon \\ Av. Rovisco Pais 1, 1049-001, Lisbon, Portugal \\ ${ }^{c}$ Department of Astronomy and Theoretical Physics \\ Lund University, Sölvegatan 14A, SE-223 62 Lund, Sweden \\ E-mail: liliana@lip.pt, andre.cordeiro@tecnico.ulisboa.pt, \\ korinna.zapp@thep.lu.se
}

Ultra-relativistic heavy-ion collisions have unlocked the study of a hot, dense state of QCD matter, the Quark-Gluon Plasma (QGP). However, due to its short lifetime, on the yoctosecond scale, the QGP must be studied with recourse to external probes, such as jets, collimated sprays of particles originated from the hard scattering. Since jets are multi-scale probes, we can use jet quenching, the collection of medium modifications of the jets' substructure, to study the evolution of medium properties at various times In this work, we show that one can assign a time structure to jets by using the formation time of a parton's emission. The obtained clustering history can be accurately reconstructed, and the medium modifications can be studied at various timescales, potentiating future tomographic measurements of the QGP. Further, by classifying jets according to the formation time of the first unclustering step, one can select, out of an inclusive measurement, jet populations that were strongly modified by the QGP. This selection of jet populations by their quenching magnitude can help to distinguish specific features of jet-QGP interaction.

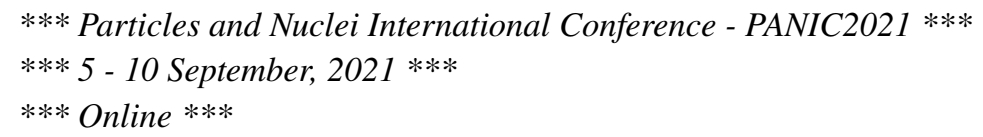

${ }^{*}$ Speaker 
Ultra-relativistic heavy ion collisions, like those at the Large Hadron Collider (LHC) and the Relativistic Heavy Ion Collider (RHIC) have allowed for the study of a hot and dense medium, characterised by the deconfinement of the parton degrees of freedom and known as the Quark-Gluon Plasma (QGP). In QGP studies, medium induced modifications of jets in heavy ion collisions, both in terms of the total jet cross-section and of their substructure, can be used to probe the Quark-Gluon Plasma within a wide range of scales.

When extracting this information from the final state particles one requires a jet definition, such that the jets' substructure resembles the QCD radiation pattern. In the case of vacuum propagated jets this is achieved by the Cambridge-Aachen (C/A) algorithm, which clusters final particles according to their angular distance, reproducing the angular ordering property of QCD emissions. However, in the presence of the QGP the phase-space for possible emissions opens up to allow anti-angular ordered showers [1], suggesting that a jet algorithm other than C/A may be better suited to jet quenching studies.

As the evolution of all medium properties is described in terms of its proper time $\tau_{\text {medium }}$, the QGP provides a time direction for the subsequent evolution of parton cascades. With this in mind, in this short report based on [2] we formulate a jet clustering algorithm ordered in splitting formation time, evaluate its performance, and explore an application for heavy ion studies.

\section{Jet Reclustering and Splitting Formation Time}

The jet algorithms under consideration consist of the generalised- $k_{T}$ family, which clusters pairs of particles with minimal distance $d_{i j}$, until all distances exceed the beam distance $d_{i B}$, as defined below,

$$
\begin{gathered}
d_{i j} \stackrel{\text { def }}{=} \min \left(p_{\mathrm{T} i}^{2 p}, p_{\mathrm{T} j}^{2 p}\right)\left(\frac{\Delta R_{i j}}{R}\right)^{2}, \\
d_{i B} \stackrel{\text { def }}{=} p_{\mathrm{T} i}^{2 p}, \quad \Delta R_{i j} \stackrel{\text { def }}{=} \sqrt{\left(\varphi_{i}-\varphi_{j}\right)^{2}+\left(y_{i}-y_{j}\right)^{2}},
\end{gathered}
$$

where the particle kinematics are given in collider variables. Here, the $p$ exponent regulates the energy dependence of the clustering distance $d_{i j}$, with the usual choices being $p=-1, p=0$, and $p=1$ for the anti- $\mathrm{k}_{\mathrm{T}}$, Cambridge-Aachen (C/A), and $\mathrm{k}_{\mathrm{T}}$ algorithms respectively. A comprehensive review of jet algorithms can be found in [3, p.14].

We also noted in [2] that by setting the algorithm exponent to $p=1 / 2$ one obtains a clustering distance which coincides with the inverse formation time of the splitting, in the soft and collinear limit,

$$
d_{12} \propto \min \left(p_{\mathrm{T} 1}, p_{\mathrm{T} 2}\right)\left(\Delta R_{12}\right)^{2} \simeq \tau_{\text {form }}^{-1}
$$

\section{Methodology}

In order to evaluate the performance of the generalised- $\mathrm{k}_{\mathrm{T}}$ algorithms three event generators with different choices of ordering variable were used. Namely, PYTHIA8 [4] using transverse 
momentum ordering, JEWEL [5] using virtual mass ordering and JEWEL with jet quenching effects, which uses an additional veto in formation time.

These event generators were set to produce dijet events at a centre-of-mass energy $\sqrt{s_{\mathrm{NN}}}=$ $5.02 \mathrm{TeV}$, and the medium effects are implemented in JEWEL through a toy model of an ideal quarkgluon gas, expanding according to a Bjorken model with $T_{\text {init }}=0.44 \mathrm{GeV}$ and $\tau_{\text {init }}=0.4 \mathrm{fm} / \mathrm{c}$.

To obtain jets from the final state hadrons, we start by reconstructing jets using the anti- $\mathrm{k}_{\mathrm{T}}$ algorithm with a radius of $R=0.5$. After identifying the leading jet in the transverse momentum region $p_{\mathrm{T}}>p_{\mathrm{T}, \min }=300 \mathrm{GeV}$ and a pseudo-rapidity in $\left|\eta_{\mathrm{jet}}\right|<1.0$, its constituent particles are reclustered using a generalised- $\mathrm{k}_{\mathrm{T}}$ algorithm for some $p$ value and a jet radius of $R=1.0$. These steps are performed within FastJet v3.3.0 [3]. Further, we found it necessary to apply the SoftDrop [6] grooming procedure to the reclustered jets, eliminating all splittings with $z_{g}<z_{\text {cut }}=0.1$.

\section{Performance of the $\tau$ Algorithm}

When quantifying the performance of the generalised- $\mathrm{k}_{\mathrm{T}}$ algorithms, we consider the distribution defined by

$$
\Delta \tau_{\text {form }} \stackrel{\text { def }}{=} \tau_{\text {form }}^{\text {PartonShower }}-\tau_{\text {form }}^{\text {Uncluster }}
$$

where $\tau_{\text {form }}^{\text {PartonShower }}$ denotes the first splitting formation time as calculated from the kinematic values read from the Monte Carlo event generators, and $\tau_{\text {form }}^{\text {Uncluster }}$ the formation time computed from the substructure of reclustered jets. Thus, a well performing reclustering algorithm is associated with $\Delta \tau_{\text {form }}$ distribution which is both narrow and centred at zero. An example of this distribution can be seen in figure 1a, pertaining to C/A reclustered jets.

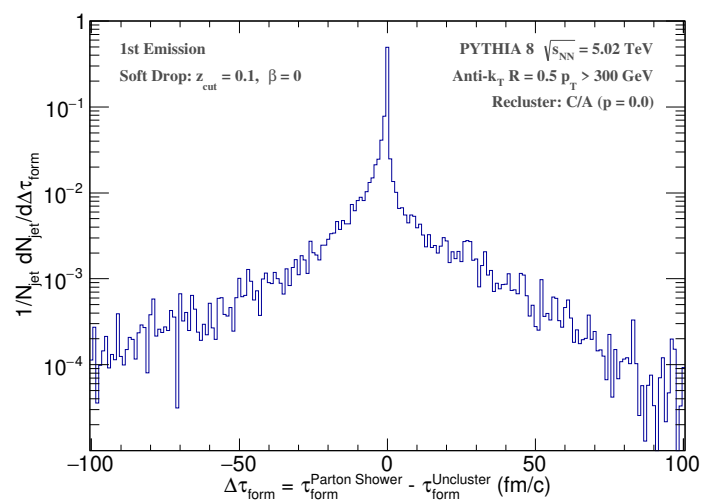

(a) $\Delta \tau_{\text {form }}$ distribution for the first emission/unclustering step with the C/A jet algorithm $(p=0)$ and $z_{\text {cut }}=0.1$.

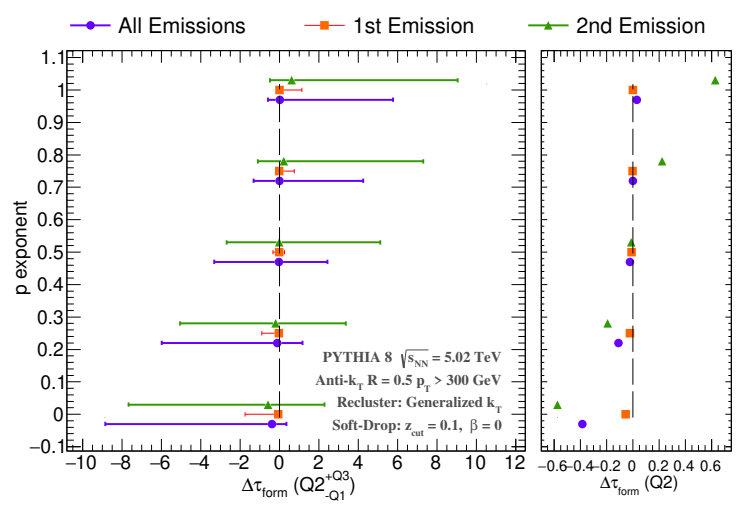

(b) Median of $\Delta \tau_{\text {form }}$ for different algorithm exponents $p$. The asymmetric error bars correspond to $+Q_{3}$ and $-Q_{1}$ quartiles. The right panel shows a zoom of $Q_{2}$ alone.

Figure 1

Due to the skewness and steepness of the $\Delta \tau_{\text {form }}$ distributions, we characterise them by their central value, defined as the median $Q_{2}$, and their width, quantified by the first and third quartiles $Q_{1}$ and $Q_{3}$. This approach allows for quantifying not only the centre and width of the distributions, but also their asymmetry. 
For PYTHIA8 samples, these values were computed for various reclustering algorithm exponents ( $p=0.0 ; 0.25 ; 0.5 ; 0.75 ; 1.0)$ and plotted in figure $1 \mathrm{~b}$. When focusing on the first emission (in orange) all considered algorithms yield $\Delta \tau_{\text {form }}$ distributions centred on zero, with central values of $p$ producing narrow and symmetric distributions. However, when considering subsequent emissions the interquartile range $\left(\mathrm{IQR} \stackrel{\text { def }}{=} Q_{3}-Q_{1}\right.$ ) becomes significant, and exponents of $p \simeq 0.5$ are required to obtain narrow and symmetric distributions.

This analysis was extended to JEWEL samples, both for vacuum and medium propagated parton cascades, yielding similar results (see [2]). Central values for the $p$ exponent continue to minimise the IQR of the $\Delta \tau_{\text {form }}$ distribution, providing the most accurate and least biased estimation of the first splitting formation time, for both vacuum and medium propagated jet samples.

\section{Jet Quenching Studies}

We now illustrate an application of the tools introduced in the previous section to heavy ion studies, by considering the nuclear modification factor, defined as the ratio between jet yields in medium and in vacuum,

$$
R_{\mathrm{AA}}\left(p_{\mathrm{T}}\right)=\frac{N_{\mathrm{evt}}^{\mathrm{pp}}}{N_{\mathrm{evt}}^{\mathrm{AA}}} \frac{\mathrm{d} N_{\mathrm{jet}}^{\mathrm{AA}} / \mathrm{d} p_{\mathrm{T}}}{\mathrm{d} N_{\mathrm{jet}}^{\mathrm{pp}} / \mathrm{d} p_{\mathrm{T}}},
$$

quantifying the jet quenching effects of the medium. This quantity is computed for the leading jets reclustered from JEWEL generated showers, while extending the kinematic region to include lower transverse momenta $\left(p_{\mathrm{T}}>100 \mathrm{GeV}\right)$. Using both $\mathrm{C} / \mathrm{A}$ and $\tau$ to recluster the leading jets we define two different jet populations, according to the formation time of their first splitting; early jets with $\tau_{\text {form }}<1 \mathrm{fm} / \mathrm{c}$, and late jets with $\tau_{\text {form }}>3 \mathrm{fm} / \mathrm{c}$. The $R_{\mathrm{AA}}\left(p_{\mathrm{T}}\right)$ of both populations is displayed in figure 2, along with those obtained from Monte Carlo values and the inclusive spectrum.

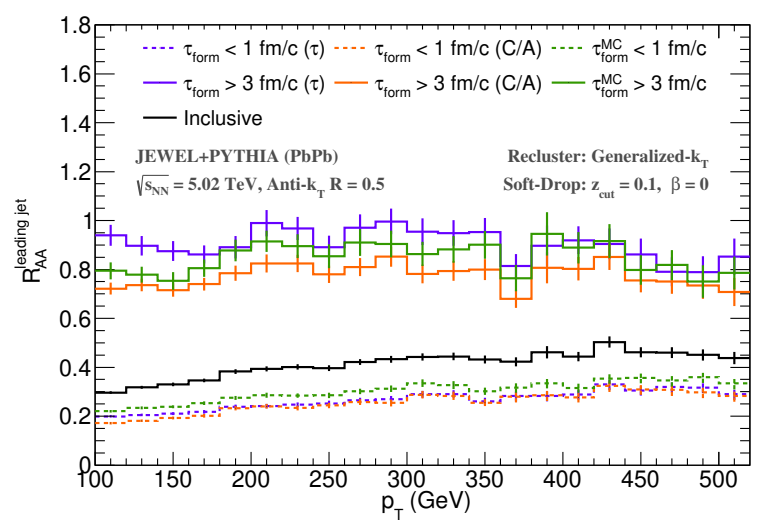

Figure 2: JEWEL $R_{\mathrm{AA}}$ of leading jets reclustered with $\tau$ (purple) or C/A (orange). The jet populations with $\tau_{\text {form }}>3 \mathrm{fm} / \mathrm{c}\left(\tau_{\text {form }}<1 \mathrm{fm} / \mathrm{c}\right)$ are shown in solid (dashed) lines. Monte Carlo results are shown in green, and the inclusive spectrum in solid black.

By comparing the $R_{\mathrm{AA}}$ for the various populations, we note a clear difference between the early and late populations relative to the full sample. Notably, the early jets are strongly suppressed, 
while the late population presents $R_{\mathrm{AA}} \sim 1$, as expected for splittings occurring after the medium has diluted considerably (energy density of $\epsilon \sim 5 \mathrm{GeV} / \mathrm{fm}^{3}$ after the first $3 \mathrm{fm}$ for the present model and settings). In the latter case, the $\tau$ algorithm appears to be a better estimator of the splitting formation time than the C/A algorithm, which underestimates the jet suppression for the late population. Ultimately, these results suggest the use of the first splitting formation time to classify jet modifications induced by the medium. This selection can provide additional constraints to the jet-medium interaction models.

\section{Summary}

We have introduced the $\tau$ jet algorithm, defined such that its clustering distance coincides with the inverse formation time $\tau_{\text {form }}^{-1}$ of the particle splittings in the soft and collinear limit. Using Monte Carlo event generators with three different orderings for parton splittings, the performance of the $\tau$ algorithm was evaluated and it was found to produce more accurate, less biased estimates of $\tau_{\text {form }}$ than C/A. Lastly, we explored an application for jet quenching studies, using the first splitting formation time as a jet quenching classifier. These results indicate that the $\tau$ algorithm may be used as a jet clustering tool for jet quenching studies in heavy-ion collisions.

\section{Acknowledgements}

AC and LA acknowledge the financial support by OE - Portugal, Fundação para a Ciência e Tecnologia (FCT) under contract DL57/2016/CP1345/CT0004 (LA) and project CERN/FISPAR/0024/2019, and by the European research Council project ERC-2018-ADG-835105 YoctoLHC. KZ acknowledges European Research Council (ERC) under the European Union's Horizon 2020 research and innovation programme (Grant agreement No. 803183 collectiveQCD).

\section{References}

[1] J. Casalderrey-Solana, Y. Mehtar-Tani, C.A. Salgado and K. Tywoniuk, New picture of jet quenching dictated by color coherence, Physics Letters B 725 (2013) 357.

[2] L. Apolinário, A. Cordeiro and K. Zapp, Time reclustering for jet quenching studies, The European Physical Journal C 81 (2021) .

[3] M. Cacciari, G.P. Salam and G. Soyez, FastJet User Manual, Eur. Phys. J. C 72 (2012) 1896 [1111.6097].

[4] T. Sjöstrand, S. Mrenna and P. Skands, A brief introduction to pythia 8.1, Computer Physics Communications 178 (2008) 852.

[5] K.C. Zapp, JEWEL 2.0.0 - Directions for Use, The European Physical Journal C 74 (2014) 2762.

[6] A.J. Larkoski, S. Marzani, G. Soyez and J. Thaler, Soft drop, Journal of High Energy Physics 2014 (2014). 